\title{
Preference Queries over Sets
}

\author{
Xi Zhang ${ }^{1}$, Jan Chomicki ${ }^{2}$ \\ Department of Computer Science and Engineering, \\ University at Buffalo, Buffalo, NY 14260, U.S.A. \\ ${ }^{1}$ xizhangeacm.org \\ ${ }^{2}$ chomickilbuffalo.edu
}

\begin{abstract}
We propose a "logic + SQL" framework for set preferences. Candidate best sets are represented using profiles consisting of scalar features. This reduces set preferences to tuple preferences over set profiles. We propose two optimization techniques: superpreference and M-relation. Superpreference targets dominated profiles. It reduces the input size by filtering out tuples not belonging to any best $k$-subset. M-relation targets repeated profiles. It consolidates tuples that are exchangeable with regard to the given set preference, and therefore avoids redundant computation of the same profile. We show the results of an experimental study that demonstrates the efficacy of the optimizations.
\end{abstract}

\section{INTRODUCTION}

In recent years, preferences have been well studied in the database and AI literature [1], [2], [3], [4], [5]. The issues addressed in this research include, among others, preference specification, preference query languages, and preference query evaluation and optimization. However, the research on preferences, with a few exceptions [6], [7], [8], has almost exclusively focused on object (or tuple) preferences which express preference relationships between individual objects or tuples in a relation.

We observe that in decision making a user sometimes needs to make a group decision based not only on the individual object properties but also on the properties of the group as a whole. For example, in university admission, the decision is made collectively on the whole incoming class. Factors such as preference for underprivileged groups are usually an important part of the decision-making process. In a board election, there might be co-existence or exclusivity relationships between candidates. A poll company usually needs to find a specific combination of candidates in order to obtain statistically representative results. Ex. 1 illustrates a book purchase scenario, where several set preferences are elaborated.

Example 1: Alice is buying three books as gifts. Here is a list of book quotes collected from different vendors:

\begin{tabular}{c|l|c|c|c}
\hline title & genre & rating & price & vendor \\
\hline$a_{1}$ & sci-fi & 5.0 & $\$ 15.00$ & Amazon \\
$a_{2}$ & biography & 4.8 & $\$ 20.00$ & $\mathrm{~B} \& \mathrm{~N}$ \\
$a_{3}$ & sci-fi & 4.5 & $\$ 25.00$ & Amazon \\
$a_{4}$ & romance & 4.4 & $\$ 10.00$ & $\mathrm{~B} \& \mathrm{~N}$ \\
$a_{5}$ & sci-fi & 4.3 & $\$ 15.00$ & Amazon \\
$a_{6}$ & romance & 4.2 & $\$ 12.00$ & $\mathrm{~B} \& \mathrm{~N}$ \\
$a_{7}$ & biography & 4.0 & $\$ 18.00$ & Amazon \\
$a_{8}$ & sci-fi & 3.5 & $\$ 18.00$ & Amazon \\
\hline
\end{tabular}

Alice needs to decide on the three books to buy. She might have any of the following preferences:

(C1) She wants to spend as little money as possible.
(C2) She prefers to get one sci-fi book.

(C3) Ideally, she prefers that all three books be from the same vendor. If that is not possible, she prefers to deal with as few vendors as possible.

In addition, Alice might have different combinations of the above preferences. For example, Alice might have both (C1) and $(\mathrm{C} 2)$, but $(\mathrm{C} 2)$ may be more important than $(\mathrm{C} 1)$ to her. This preference is expressed as follows:

(C4) Alice's preference is a prioritized composition of (C2) and $(\mathrm{C} 1)$.

The preference $(\mathrm{C} 1)$ can be directly simulated by a tuple preference over Book, such that for any $t_{1}, t_{2} \in B o o k, t_{1}$ is preferred to $t_{2}$ iff $t_{1}$.price $<t_{2}$.price. Then the top three books in Book (according to this preference) constitute the best answer set. However, in the other cases, i.e., (C2-C4), such a simulation is not possible.

Our goal is to develop a general framework for set preferences from the database perspective. Previous work on set preferences was predominantly done in AI research [7], [8]. To the best of our knowledge, the only work that addresses a related problem in the database context is [6]. However, the form of set preferences which can be expressed by the language in [6] is quite restricted. In this work, we emphasize the generality of preferences by providing a general formal framework for set preferences which combines first-order logic and SQL. We work with sets of fixed cardinality to focus on the composition of sets. Preferences involving fixed-cardinality sets emerge in many real applications, as outlined in Sec. V.

We observe that a large class of set preferences has two components: (1) Quantities of interest; (2) Desired value or order of those quantities.

\begin{tabular}{l|l|c}
\multicolumn{2}{c|}{ Example 2: In Ex. 1, } \\
\hline & Quantity of Interest & $\begin{array}{c}\text { Desired Value } \\
\text { or Order }\end{array}$ \\
\hline (C1) & total cost & $<$ \\
(C2) & number of sci-fi books & 1 \\
(C3) & number of distinct vendors & $<$ \\
\hline
\end{tabular}

Consequently, our framework consists of two components: (1) profiles: tuples of features, each feature capturing a quantity of interest; (2) profile preference relations to specify desired values or orders.

The main idea is to construct the profiles of candidate subsets based on their features. Since each profile is a tuple of features, the original set preference can now be formulated as a tuple preference over the profiles. Moreover, the best subsets 
under the set preference in the original relation correspond to the best profiles.

Our major contributions are:

- We propose a formal framework, combining SQL and first-order logic, for the specification of (second-order) set preferences via profiling.

- We define the notion of superpreference relation which helps to prune candidate best subsets. We show how to systematically construct first-order definitions of superpreference relations in restricted cases.

- We define the notion of $M$-relation that helps to consolidate candidate subsets. We show how to compute optimal M-relations using SQL.

- We report on an experimental study which demonstrates the efficacy of our optimization techniques and their synergistic character.

The rest of the paper is organized as follows. Sec. II provides the basic notions used throughout this work. We first elaborate our framework in Sec. III and Sec. IV, and then discuss the computational issues involved in computing the best subsets in Sec. V-A. We describe efficient optimizations to significantly reduce the computation effort in Sec. V-B (superpreference) and Sec. V-C (M-relation). In Sec. V-E, we show how to combine those two optimizations. We report on an empirical study of their performance in Sec. VI. Finally, we discuss the related work in Sec. VII and conclude in Sec. VIII.

\section{BASIC NOTIONS}

A binary relation $>$ is irreflexive iff $\forall x . x \ngtr x$. It is transitive iff $\forall x, y, z .(x>y \wedge y>z) \Rightarrow x>z$. It is negatively transitive iff $\forall x, y, z .(x \ngtr y \wedge y \ngtr z) \Rightarrow x \ngtr z$. It is connected iff $\forall x, y . x>y \vee y>x \vee x=y$. A strict partial order (SPO) is an irreflexive, transitive binary relation. A weak order (WO) is a negatively transitive SPO. A total order (TO) is a connected SPO. SPO conditions are generally considered to capture rationality of preferences. WOs arise when preferences are defined using a numeric scoring function. SPOs are more general than WOs, for example skyline, pskyline and many other kinds of preferences [1], [2], [9] are SPOs but not WOs.

A multiset is a generalization of a set. In a multiset, each member may have more than one membership, in contrast to only one membership in a set. The cardinality of a set (multiset) $s$, denoted by $|s|$, is the total number of elements in $s$. A set (multiset) $s^{\prime}$ is a subset (multisubset) of the set (multiset) $s$ iff each member of $s^{\prime}$ is also a member of $s$. For example, assume multisets $s_{1}=\{a, b, b\}, s_{2}=\{a, b\}, s_{3}=$ $\{a, a, b\}$. Then, $\left|s_{1}\right|=3,\left|s_{2}\right|=2$ and $\left|s_{3}\right|=3$, respectively. Furthermore, $s_{2} \subseteq s_{1}$, but $s_{3} \nsubseteq s_{1}$.

We make the standard assumptions of the relational model of data. In particular, we assume that we have two attribute domains: rational numbers $(\mathcal{Q})$ and uninterpreted constants $(\mathcal{D})$. For a relation schema $R=\left\langle A_{1}, \ldots, A_{m}\right\rangle$, we define the domain of $R$ as the cross product of the domains of its attributes, i.e. $\operatorname{Dom}(R)=\operatorname{Dom}\left(A_{1}\right) \times \ldots \times \operatorname{Dom}\left(A_{m}\right)$.
Definition 1: Tuple Preference [2], [4]. Given a relation schema $R=\left\langle A_{1}, \ldots, A_{m}\right\rangle$, a tuple preference relation $\rangle$ over $R$ is a subset of $[\operatorname{Dom}(R)]^{2}$. If for a first order formula $C, C\left(t_{1}, t_{2}\right) \Leftrightarrow t_{1}>t_{2}$, then the tuple preference is defined by the formula $C$. We denote the preference relation by $>_{C}$.

For a tuple preference, the computation of the best tuples is embedded into Relational Algebra (RA) in the form of the winnow operator. It is commonly assumed that the tuple preference is an SPO.

Definition 2: Winnow Operator [4]. If $R$ is a relation schema and $>_{C}$ a preference relation over $R$, then the winnow operator is written as $\omega_{C}(R)$, and for every instance $r$ of $R$ : $\omega_{C}(r)=\left\{t \in r \mid \nexists t^{\prime} \in r . t^{\prime}>_{C} t\right\}$.

Tuples in $\omega_{C}(r)$ are not dominated by other tuples in $r$, and are thus the best tuples of $r$. Denote by subsets $(r)$ the power set of the relation $r$. We capture the quantities of interest for subsets using subset features.

Definition 3: Subset Feature. Given a relation $r$, a subset feature $\mathcal{F}$ is a function which maps the subsets of $r$ to exactly one of the two attribute domains. Denote by $\operatorname{Dom}(\mathcal{F})$ the domain of $\mathcal{F}$, either $\mathcal{Q}$ or $\mathcal{D}$.

Definition 4: Subset Profile Schema. Given a relation $r$, a subset profile schema $\Gamma$ is a schema $\left\langle\mathcal{F}_{1}, \ldots, \mathcal{F}_{m}\right\rangle$, where $\mathcal{F}_{i}$ is a subset feature, $i=1, \ldots, m$.

Definition 5: Subset Profile Relation. Given a relation $r$ and its subset profile schema $\Gamma=\left\langle\mathcal{F}_{1}, \ldots, \mathcal{F}_{m}\right\rangle$, the subset profile relation $\gamma$ is defined as

$$
\gamma=\left\{\left\langle\mathcal{F}_{1}(s), \ldots, \mathcal{F}_{m}(s)\right\rangle \mid s \in \operatorname{subsets}(r)\right\}
$$

The tuple $\left\langle\mathcal{F}_{1}(s), \ldots, \mathcal{F}_{m}(s)\right\rangle$ is the profile of $s$ under $\Gamma$, denoted by profile $_{\Gamma}(s)$.

When the context is unambiguous, we omit subset, and refer to the above concepts as feature, profile schema and profile relation, respectively.

\section{AgGregate Features}

In this work, without loss of generality, we consider singlevalued features whose values are rational numbers, as it is often the case in real applications [7]. This is achieved by defining features as aggregate values in Def. 6. Other possible single-valued features include boolean features, which will be discussed in Sec. VII.

Definition 6: Aggregate Subset Features. Given a relation $r$ with a schema $R$, an aggregate subset feature $\mathcal{F}$ is defined by a parameterized SQL query of the form

SELECT expr FROM \$S WHERE condition where: (1) $\$ S$ is a distinguished set parameter whose values can be instantiated to an arbitrary subset of $r$, i.e. $\operatorname{Dom}(\$ \mathrm{~S}) \subseteq$ $\operatorname{subsets}(r)$. (2) expr is of the form aggr ([DISTINCT] A) where $\operatorname{agg} r \in\{\min , \max$, sum, count, $\operatorname{avg}\}, A$ is an attribute of $R$, or a function of constants and the above aggregates. (3) FROM list contains a single item $\$ S$ or an alias for $\$ S$. (4) WHERE clause is a conjunction of atomic comparisons. As we will see, the cardinality of $\$ S$ can be restricted.

Example 3: In Ex. 1, the quantity of interest in (C1), (C2) and (C3) is captured by the subset feature $\mathcal{F}_{1}, \mathcal{F}_{2}$ and $\mathcal{F}_{3}$, respectively. 


$$
\begin{aligned}
& \mathcal{F}_{1} \equiv \text { SELECT } \text { sum(price) FROM } \$ \text { S } \\
& \mathcal{F}_{2} \equiv \text { SELECT count (title) FROM } \$ S \\
& \text { WHERE genre=' SCi-fi' } \\
& \mathcal{F}_{3} \equiv \text { SELECT count (DISTINCT vendor) FROM \$S }
\end{aligned}
$$
where $\$ S$ is a set parameter that can be substituted by any three-element subset of Book, as Alice decides to buy three books. Given any subset $s$ of Book, we can evaluate the value of each feature by instantiating the set parameter $\$ S$ in the feature definition with $s$. For example, assume $s=\left\{a_{1}, a_{2}, a_{3}\right\}$, then $\mathcal{F}_{1}(s)$ is the scalar result of the query SELECT sum(price) FROM $s$, which is $\$ 15.00+\$ 20.00+\$ 25.00=\$ 60.00$. Similarly, $\mathcal{F}_{2}(s)=2$ due to the result of SELECT count (title) FROM $s$ WHERE genre='sci-fi', and $\mathcal{F}_{3}(s)=2$ due to that of SELECT count (DISTINCT vendor) FROM s. Let the subset profile schema $\Gamma=\left\langle\mathcal{F}_{1}, \mathcal{F}_{2}\right\rangle$. The subset profile relation $\gamma$ (corresponding to Book) contains, among others, the following tuples: $(\$ 60,2)$, which is the profile of the subsets $\left\{a_{1}, a_{2}, a_{3}\right\}$ and $\left\{a_{2}, a_{3}, a_{5}\right\}$; and $(\$ 61,2)$, which is the profile of $\left\{a_{3}, a_{7}, a_{8}\right\}$.

\section{Profile-BAsed Set Preferences}

Now we can define set preferences over subsets as tuple preferences over the corresponding profiles. Commonly, a tuple preference relation is defined using a first-order formula [4], as is the case for the tuple preference simulating (C1) in Ex. 1 .

Definition 7: Set Preference. Given a relation schema $R=$ $\left\langle A_{1}, \ldots, A_{m}\right\rangle$, a set preference relation $\gg$ is a finite subset of the product $[\operatorname{subsets}(\operatorname{Dom}(R))]^{2}$.

In principle, set preferences could also be defined using logic formulas. However, second-order variables would be necessary. To avoid the conceptual and computational complexity associated with such variables, we consider only set preferences that are based on profile preferences.

Definition 8: Profile-based Set Preference. Let $\Gamma=$ $\left\langle\mathcal{F}_{1}, \ldots, \mathcal{F}_{m}\right\rangle$ be a profile schema and $\rangle_{C}$ a tuple preference relation, which is a subset of $\left[\operatorname{Dom}\left(\mathcal{F}_{1}\right) \times \ldots \times \operatorname{Dom}\left(\mathcal{F}_{m}\right)\right]^{2}$. For every sets $s_{1}$ and $s_{2}$,

$$
s_{1} \gg_{(\Gamma, C)} s_{2} \Leftrightarrow \text { profile }_{\Gamma}\left(s_{1}\right)>_{C} \text { profile }_{\Gamma}\left(s_{2}\right) .
$$

We say that the set $s_{1}$ is preferred to the set $s_{2}$ and the profilebased set preference relation is denoted by $\gg_{(\Gamma, C)}$.

Proposition 4.1: If $>_{C}$ is an SPO, then for any profile schema $\Gamma$, the set preference relation $\gg_{(\Gamma, C)}$ is an SPO, too.

Recall that essential components of set preferences are the desired values or orders of the quantities of interest, which are captured by a preference relation over profiles. In fact, in order to elaborate a set preference in our framework, a user needs to do the following: (1) Provide a subset profile schema by defining subset features $\mathcal{F}_{1}, \ldots, \mathcal{F}_{m}$. (2) Specify the profile preference using a tuple preference formula.

Def. 8 provides a general framework for set preferences. For the reasons we will shortly discuss in Sec. V, we restrict our interest to set preferences among subsets of fixed cardinality. Here, we only point out that in Ex. 1, Alice buys three books, and thus we work with subsets of fixed cardinality 3 .

Example 4: Assume the profile schema $\Gamma=\left\langle\mathcal{F}_{1}, \mathcal{F}_{2}, \mathcal{F}_{3}\right\rangle$ as in Ex. 3. We define the preference formula $C i,(i=1,2,3)$ over $\Gamma$, such that the individual set preference $(\mathrm{Ci}), i=1,2,3$ is based on $\Gamma$ and $>_{C i}$. For example,

$$
\begin{aligned}
& s_{1} \gg_{(\Gamma, C 1)} s_{2} \\
& \Leftrightarrow\left\langle\mathcal{F}_{1}\left(s_{1}\right), \mathcal{F}_{2}\left(s_{1}\right), \mathcal{F}_{3}\left(s_{1}\right)\right\rangle>_{C 1}\left\langle\mathcal{F}_{1}\left(s_{2}\right), \mathcal{F}_{2}\left(s_{2}\right), \mathcal{F}_{3}\left(s_{2}\right)\right\rangle \\
& \Leftrightarrow \mathcal{F}_{1}\left(s_{1}\right)<\mathcal{F}_{1}\left(s_{2}\right) .
\end{aligned}
$$

Individual preference formulas can be the building blocks of more complicated preferences, where formulas are assembled to express union, intersection, prioritized composition and Pareto composition of preferences [4], [2].

Example 5: Consider (C4) in Ex. 1, i.e. the prioritized composition of $(\mathrm{C} 2)$ and $(\mathrm{C} 1)$. Let the profile schema $\Gamma=\left\langle\mathcal{F}_{1}, \mathcal{F}_{2}, \mathcal{F}_{3}\right\rangle$, and the preference formula $C 4$ over $\Gamma$ be the prioritized composition [4] of the preference formulas $C 2$ and $C 1$

$$
\begin{aligned}
s_{1} \gg_{(\Gamma, C 4)} s_{2} & \Leftrightarrow\left(\mathcal{F}_{2}\left(s_{1}\right)=1 \wedge \mathcal{F}_{2}\left(s_{2}\right) \neq 1\right) \\
& \vee\left(\mathcal{F}_{2}\left(s_{1}\right)=1 \wedge \mathcal{F}_{2}\left(s_{2}\right)=1 \wedge \mathcal{F}_{1}\left(s_{1}\right)<\mathcal{F}_{1}\left(s_{2}\right)\right) \\
& \vee\left(\mathcal{F}_{2}\left(s_{1}\right) \neq 1 \wedge \mathcal{F}_{2}\left(s_{2}\right) \neq 1 \wedge \mathcal{F}_{1}\left(s_{1}\right)<\mathcal{F}_{1}\left(s_{2}\right)\right) .
\end{aligned}
$$

We see that the preference formula $C 4$ expresses precisely the set preference $(\mathrm{C} 4)$.

\section{Computing the Best $k$-Subsets}

Recall that we make a decision to work with subsets of fixed cardinality. There are two reasons for this choice. First, fixed cardinality allows the user to focus on the composition of a best subset and the interactions between the tuples in it. Some set properties might have an inherent bias towards sets of certain cardinalities. For example, without limiting the cardinality in the set preference $(\mathrm{C} 1)$, the user certainly prefers small subsets, since buying fewer books costs less. In the extreme case, the best subset would be the empty set, with cost $\$ 0$. In reality, this is rarely what the user intends. Likewise, many applications have an explicit or implicit requirement on cardinality. For example, a board election typically has a fixed number of seats to be filled. A university usually admits a class of a predetermined size. A poll company has limited resources for interviewing only a certain number of people.

Definition 9: $k$-subset. Given a relation $r$ and a positive integer $k, k \leqslant|r|$, a $k$-subset $s$ of $r$ is a subset of $r$ with cardinality $k$, i.e. $s \subseteq r$ and $|s|=k$. Denote by $k$-subsets $(r)$ the set of all $k$-subsets of $r$.

Definition 10: $k$-subset Profile Relation. Given a relation $r$ and its subset profile schema $\Gamma=\left\langle\mathcal{F}_{1}, \ldots, \mathcal{F}_{m}\right\rangle$, the $k$-subset profile relation $\gamma_{k}$ is defined as

$$
\gamma_{k}=\left\{\left\langle\mathcal{F}_{1}(s), \ldots, \mathcal{F}_{m}(s)\right\rangle \mid s \in k \text {-subsets }(r)\right\}
$$

We omit the subscript $k$ in $\gamma_{k}$ when the context is unambiguous.

\section{A. Basic Algorithm}

For a tuple preference, the computation of the best tuples is embedded into Relational Algebra (RA) in the form of a winnow operator (c.f., Def. 2). In addition to the universal Nested Loops (NL) algorithm, several other efficient evaluation algorithms for winnow have been proposed when the preference relation is an SPO, among others, Block Nested Loops (BNL) [1] and Sort-Filter-Skyline (SFS) [10]. In our framework, a set preference relation is formulated as a tuple preference relation $>_{C}$ over a profile schema $\Gamma$. Then, a 
winnow operator is defined over $\Gamma$, i.e. $\omega_{C}(\Gamma)$. The best $k$ subsets are computed by winnowing over the profile relation $\gamma$ containing the profiles of all $k$-subsets of a given relation $r$. Alg. 1 applies winnow on a stream of profiles of all $k$-subsets.

Algorithm 1 (NAIVE) Basic Algorithm
Require: a profile schema $\Gamma$, an SPO profile preference
relation $>_{C}$, a relation $r$ and a positive integer $k, k<|r|$
Ensure: the best $k$-subsets of $r$ under the set pref. $\rangle_{(\Gamma, C)}$
1: Generate all $k$-subsets of relation $r$ and compute the
profiles of each $k$-subset based on the schema $\Gamma$, obtaining
the profile relation $\gamma$.
2: Compute $\gamma^{\prime}=\omega_{C}(\gamma)$ using any winnow evaluation
algorithm, e.g. BNL [1].
3: Retrieve the subsets corresponding to the profiles in $\gamma^{\prime}$.

For the generation of candidate $k$-subsets in Line 1 of Alg. 1, any sound and complete $k$-subset generator suffices. We arbitrarily choose a lexicographical $k$-subset generator [11] which produces $k$-subsets in the lexicographical order of the tuple indices.

Alg. 1 is suitable for a small $k$, while for a large $k$, the number of $k$-subsets $\left(\begin{array}{l}n \\ k\end{array}\right)$ can be intimidating, and exhaustive enumeration might not be acceptable. On the other hand, since the number of best sets can be as large as $\left(\begin{array}{l}n \\ k\end{array}\right)$ when the set preference relation $\gg_{(\Gamma, C)}$ is empty, the worst case complexity $\Omega\left(n^{k}\right)$ is unavoidable. In the following sections, we identify redundant $k$-subsets generated by the basic algorithm, i.e., $k$ subsets whose profiles will be dominated by other profiles or $k$-subsets whose profiles are repeated. We propose two optimization techniques: superpreference and M-relation. Roughly speaking, superpreference targets the dominated profiles. It filters out tuples that do not contribute to any best $k$-subset. $M$ relation targets repeated profiles. It groups together tuples that are exchangeable with regard to the given set preference, and therefore avoids redundant computation of the same profile. Both techniques tend to reduce the number of candidate $k$ subsets and therefore speed up the computation of the best subsets.

\section{B. Superpreference}

The idea is that, given a set preference relation $\gg_{(\Gamma, C)}$, we are trying to find a superpreference relation $>^{+}$such that if $t_{1}>^{+} t_{2}$, then every $k$-subset with $t_{1}$ is preferred (under $\left.\gg_{(\Gamma, C)}\right)$ to every $k$-subset with $t_{2}$ as long as these two $k$ subsets are otherwise identical.

Definition 11: Superpreference Relation. Given a relation $r$, a positive integer $k \leqslant|r|$ and a set preference relation $\gg_{(\Gamma, C)}$, the corresponding superpreference relation, denoted by $>^{+}$, is such that

$t_{1}>^{+} t_{2} \Leftrightarrow \quad t_{1} \in r \wedge t_{2} \in r \wedge\left[\forall s^{\prime} \in(k-1)\right.$-subsets $\left(r \backslash\left\{t_{1}, t_{2}\right\}\right)$, $\left.s^{\prime} \cup\left\{t_{1}\right\} \gg_{(\Gamma, C)} s^{\prime} \cup\left\{t_{2}\right\}\right]$.

The cover of $t$ is the set of tuples dominating $t$ under $>^{+}$, i.e. cover $(t)=\left\{t^{\prime} \in r \mid t^{\prime}>^{+} t\right\}$. Whenever $t_{1}>^{+} t_{2} \Leftrightarrow t_{1} \in$ $r \wedge t_{2} \in r \wedge C^{+}\left(t_{1}, t_{2}\right)$ and $C^{+}$is a first-order formula, we say that $>^{+}$corresponds to $C^{+}$.

Proposition 5.1: Given a relation $r$, a positive integer $k<|r|$ and a set preference relation $\gg_{(\Gamma, C)}$, for every $s \in k-\operatorname{subsets}(r)$,

$$
\left[\nexists s^{\prime} \in k \text {-subsets }(r), s^{\prime} \gg_{(\Gamma, C)} s\right] \Rightarrow[\forall t \in s, \operatorname{cover}(t) \subseteq s] .
$$

Eqn. (1) states that if a $k$-subset $s$ is not dominated by any other $k$-subset, then $s$ should contain the cover of each member. It is a necessary condition for a best $k$-subset. Prop. 5.1 enables two optimizations in Alg. 2: (1) Every tuple $t$ whose $|\operatorname{cover}(t)| \geqslant k$ is discarded, as it cannot belong to any best $k$-subset (Line 2); (2) During the candidate $k$-subset generation in Line 3, we skip over those candidate $k$-subsets not leading to a best $k$-subset according to Prop. 5.1. To be more specific, in Line 3 of Alg. 2, we use a modified version of the standard lexicographical $k$-subset generator, which applies a filter when enumerating $k$-subsets. It returns the first successive $k$-subset satisfying Eqn. (1) if any.

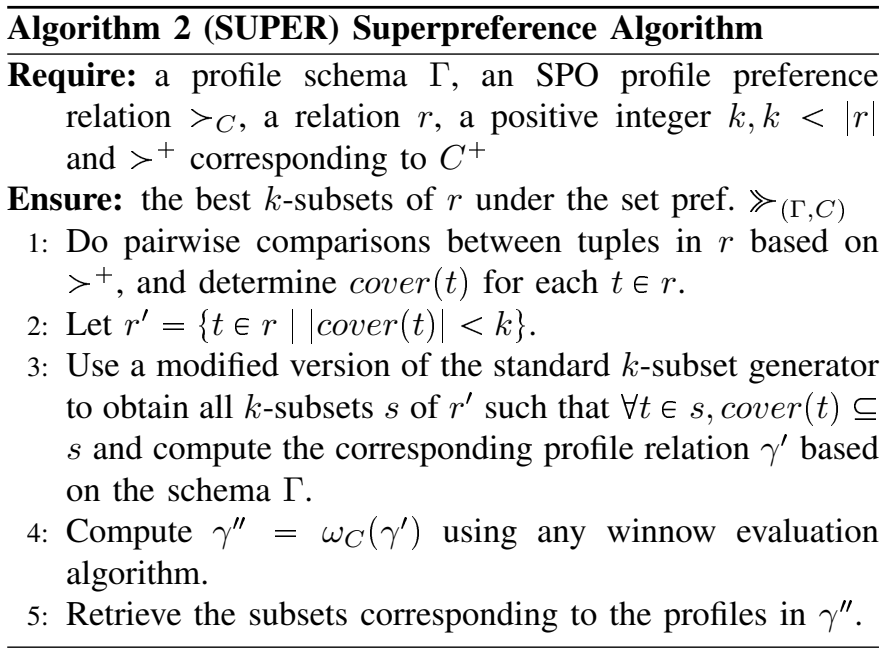

\section{Algorithm 3 Superpreference Algorithm under Weak Or- der Superpreference}

Require: a profile schema $\Gamma$, a WO profile preference relation $>_{C}$, a relation $r$, a positive integer $k, k<|r|$ and $>^{+}$ corresponding to $C^{+}$

Ensure: the best $k$-subsets of $r$ under the set pref. $\gg_{(\Gamma, C)}$

1: Let $r^{\prime}=\omega_{C^{+}}(r)$.

2: If $\left|r^{\prime}\right| \geqslant k$, generate all $k$-subsets of $r^{\prime}$ and the corresponding profile relation $\gamma^{\prime}$ based on the schema $\Gamma$, otherwise $r^{\prime}=r^{\prime} \cup \omega_{C^{+}}\left(r-r^{\prime}\right)$ and repeat this step.

3: Compute $\gamma^{\prime \prime}=\omega_{C}\left(\gamma^{\prime}\right)$ using any winnow evaluation algorithm.

4: Retrieve the subsets corresponding to the profiles in $\gamma^{\prime \prime}$.

If the superpreference $>^{+}$is a WO, Alg. 3 can further reduce the input $\left(r^{\prime}\right)$ to the lexicographical $k$-subset generator, which leads to fewer candidate $k$-subsets. In order to illustrate the importance of the WO requirement in Alg. 3, let $r_{1}=\omega_{C^{+}}(r), r_{2}=\omega_{C^{+}}\left(r-r_{1}\right), r_{3}=\omega_{C^{+}}\left(r-r_{1}-r_{2}\right) \ldots$, until all tuples in $r$ are exhausted. Let $\cup r_{i}=r_{1} \cup \ldots \cup r_{i}$, then $r_{i}=\omega_{C^{+}}\left(r-\cup r_{i-1}\right)$. If the superpreference $>^{+}$is a WO, then by the definition of WO every tuple in $\cup r_{i}$ is superpreferred to every tuple in $r-\cup r_{i}$. In other words, every tuple in $\cup r_{i}$ belongs to the cover of every tuple in $r-\cup r_{i}$. If $\cup r_{i}$ contains 
at least $k$ tuples, we know that the cardinality of the cover of every tuple in $r-\cup r_{i}$ is at least $k$, and thus the tuple can be discarded. A general SPO does not guarantee such a relationship and thus we have to keep track of the covers of individual tuples (Alg. 2).

It still remains to show how to construct the formula $C^{+}$ given a profile schema $\Gamma$ and a profile preference formula $C$. We show below that for restricted classes of profile schemas and profile preference formulas, $C^{+}$can be constructed systematically. Def. 12 introduces an important class of features, namely additive features. As we will present shortly in this section as well as in Sec. V-C, additivity of features enables various optimization techniques.

Definition 12: Additive Subset Features. Given a relation $r$ and a subset feature $\mathcal{F}, \mathcal{F}$ is additive iff (1) for every tuple $t \in r, \mathcal{F}(\{t\})=f(t)$, and (2) for every $s \in \operatorname{subsets}(r)$ and every $t \in r-s, \mathcal{F}(s \cup\{t\})=\mathcal{F}(s)+f(t)$, where $f$ is a function of $t$ only, called the base of $\mathcal{F}$.

Proposition 5.2: If an aggregate feature $\mathcal{F}$ is of the form SELECT expr FROM \$S WHERE simple-condition where: (1) expr is of the form aggr(A), where aggr $\in$ \{sum, count \} and $\mathrm{A}$ is an attribute of the schema of $r$, or a linear combination of constants and the above aggregates; (2) simple-condition does not contain subqueries, then $\mathcal{F}$ is additive.

Theorem 1: If $k<|r|-1$ is the chosen cardinality and a profile-based set preference is defined as a DNF formula of the following form

$$
s_{1} \gg_{(\Gamma, C)} s_{2} \Leftrightarrow \bigvee_{i=1}^{n}\left(\bigwedge_{j=1}^{m_{i}}\left(\mathcal{F}_{i j}\left(s_{1}\right) \theta \mathcal{F}_{i j}\left(s_{2}\right)\right)\right)
$$

where $\theta \in\{=, \neq,<,>, \leqslant, \geqslant\}$ and $\mathcal{F}_{i j}$ is an additive aggregate subset feature, then $C^{+}$is a first-order formula independent of $k$.

Proof: For every $i$ and $j$, we have the rewriting

$$
\begin{aligned}
& t_{1}>^{+} t_{2} \Leftrightarrow \quad t_{1} \in r \wedge t_{2} \in r \wedge\left[\forall s^{\prime} \in(k-1) \text {-subsets }\left(r \backslash\left\{t_{1}, t_{2}\right\}\right),\right. \\
&\left.\quad s^{\prime} \cup\left\{t_{1}\right\} \gg(\Gamma, C) s^{\prime} \cup\left\{t_{2}\right\}\right] \\
& \Leftrightarrow \quad t_{1} \in r \wedge t_{2} \in r \wedge\left[\forall s^{\prime} \in(k-1) \text {-subsets }\left(r \backslash\left\{t_{1}, t_{2}\right\}\right),\right. \\
&\left.\bigvee_{i=1}^{n}\left(\bigwedge_{j=1}^{m_{i}}\left(\mathcal{F}_{i j}\left(s^{\prime} \cup\left\{t_{1}\right\}\right) \theta \mathcal{F}_{i j}\left(s^{\prime} \cup\left\{t_{2}\right\}\right)\right)\right)\right]
\end{aligned}
$$

Since $\mathcal{F}_{i j}$ is additive, we can show by case study that each $\mathcal{F}_{i j}\left(s^{\prime} \cup\left\{t_{1}\right\}\right) \theta \mathcal{F}_{i j}\left(s^{\prime} \cup\left\{t_{2}\right\}\right)$ is equivalent to a formula $D_{i j}\left(t_{1}\right.$, $t_{2}$ ) of $t_{1}$ and $t_{2}$ only. For example, assume aggr in $\mathcal{F}_{i j}$ is sum, and $\theta$ is $>$, then with the abuse of the indicator function $c_{i j}(\cdot)$ as a boolean variable, we have

$\mathcal{F}_{i j}\left(s^{\prime} \cup\left\{t_{1}\right\}\right)>\mathcal{F}_{i j}\left(s^{\prime} \cup\left\{t_{2}\right\}\right)$

$\Leftrightarrow \mathcal{F}_{i j}\left(s^{\prime}\right)+c_{i j}\left(t_{1}\right) \cdot t_{1} \cdot A_{i j}>\mathcal{F}_{i j}\left(s^{\prime}\right)+c_{i j}\left(t_{2}\right) \cdot t_{2} \cdot A_{i j}$

$\Leftrightarrow \quad\left(c_{i j}\left(t_{1}\right) \wedge c_{i j}\left(t_{2}\right) \wedge t_{1} . A_{i j}>t_{2} . A_{i j}\right)$

$\vee\left(c_{i j}\left(t_{1}\right) \wedge \neg c_{i j}\left(t_{2}\right) \wedge t_{1} \cdot A_{i j}>0\right)$

$\vee\left(\neg c_{i j}\left(t_{1}\right) \wedge c_{i j}\left(t_{2}\right) \wedge t_{2} . A_{i j}<0\right)$

where $c_{i j}(t)$ indicates whether the tuple $t$ satisfies the WHERE condition of the feature $\mathcal{F}_{i j}$. Therefore,

$t_{1}>^{+} t_{2} \Leftrightarrow \quad t_{1} \in r \wedge t_{2} \in r \wedge\left[\forall s^{\prime} \in(k-1)\right.$-subsets $\left(r \backslash\left\{t_{1}, t_{2}\right\}\right)$,

$$
\left.\bigvee_{i=1}^{n}\left(\bigwedge_{j=1}^{m_{i}}\left(D_{i j}\left(t_{1}, t_{2}\right)\right)\right)\right]
$$

where $D_{i j}\left(t_{1}, t_{2}\right)$ is a formula with only variables $t_{1}$ and $t_{2}$. In particular, $D_{i j}\left(t_{1}, t_{2}\right)$ does not contain the set variable $s^{\prime}$. By rewriting every conjunct in $C$, we have

$$
t_{1}>^{+} t_{2} \Leftrightarrow t_{1} \in r \wedge t_{2} \in r \wedge \bigvee_{i=1}^{n}\left(\bigwedge_{j=1}^{m_{i}}\left(D_{i j}\left(t_{1}, t_{2}\right)\right)\right),
$$

and thus $C^{+}\left(t_{1}, t_{2}\right)=\bigvee_{i=1}^{n}\left(\bigwedge_{j=1}^{m_{i}}\left(D_{i j}\left(t_{1}, t_{2}\right)\right)\right)$.

The additive subset features identified in Prop. 5.2 are eligible for the rewriting technique in Thm. 1. However, this rewriting does not work for non-additive features defined by min, or max, or avg with non-TRUE WHERE condition. In those cases, if we rewrite $\mathcal{F}(s)$ as an expression of $s^{\prime}$ and $t$, the term(s) containing variable $s^{\prime}$ cannot be canceled on both sides of $\theta$. Intuitively, it states that we cannot determine which of $t_{1}$ and $t_{2}$ is superpreferred without looking at the tuples in $s^{\prime}$. For example, consider the case where aggr is avg, the condition is non-TRUE, and $\theta$ is $>$, the rewriting technique in Thm. 1 generates the following inequality:

$$
\begin{aligned}
& \mathcal{F}_{i j}\left(s^{\prime} \cup\left\{t_{1}\right\}\right)>\mathcal{F}_{i j}\left(s^{\prime} \cup\left\{t_{2}\right\}\right) \Leftrightarrow \\
& \frac{\left(b_{i j}\left(s^{\prime}\right) \cdot \mathcal{F}_{i j}\left(s^{\prime}\right)+c_{i j}\left(t_{1}\right) \cdot t_{1} \cdot A_{i j}\right)}{\left(b_{i j}\left(s^{\prime}\right)+c_{i j}\left(t_{1}\right)\right)}>\frac{\left(b_{i j}\left(s^{\prime}\right) \cdot \mathcal{F}_{i j}\left(s^{\prime}\right)+c_{i j}\left(t_{2}\right) \cdot t_{2} \cdot A_{i j}\right)}{\left(b_{i j}\left(s^{\prime}\right)+c_{i j}\left(t_{2}\right)\right)},
\end{aligned}
$$

where $b_{i j}\left(s^{\prime}\right)=\left|\left\{t \mid t \in s^{\prime} \wedge c_{i j}(t)\right\}\right|$. After simplifying the above inequality, we still have terms of variable $s^{\prime}$.

When Thm. 1 is applicable, we can often use domain knowledge to significantly simplify the rewriting in Thm. 1 . For the rewriting example in the proof of Thm. 1, if $A_{i j}$ is price, which is always positive, then the rewriting result can be simplified to $c_{i j}\left(t_{1}\right) \wedge\left(t_{1} . A_{i j}>t_{2} . A_{i j} \vee \neg c_{i j}\left(t_{2}\right)\right)$.

Example 6: In Ex. 1, consider the following preference: (C5) Alice wants to spend as little money as possible on sci-fi books. (C6) Alice wants the total rating of books to be as high as possible. The set preference is the intersection of (C5) and (C6). Therefore, we have $\Gamma=\left\langle\mathcal{F}_{5}, \mathcal{F}_{6}\right\rangle$,

$\mathcal{F}_{5} \equiv$ SELECT sum(price) FROM $\$ S$

WHERE genre='sci-fi'

$\mathcal{F}_{6} \equiv$ SELECT $\operatorname{sum}($ rating) FROM $\$$ S

and $s_{1} \gg_{(\Gamma, C)} s_{2}$ iff $\mathcal{F}_{5}\left(s_{1}\right)<\mathcal{F}_{5}\left(s_{2}\right) \wedge \mathcal{F}_{6}\left(s_{1}\right)>\mathcal{F}_{6}\left(s_{2}\right)$. The superpreference formula $C^{+}$obtained under the assumption that price $>0$ is

$$
\begin{aligned}
C^{+}\left(t_{1}, t_{2}\right) \Leftrightarrow & t_{1} \text {.rating }>t_{2} . \text { rating } \wedge t_{2} . \text { genre }=\text { 'sci-fi' } \\
& \wedge\left(t_{1} . \text { price }<t_{2} . \text { price } \vee t_{1} . \text { genre } \neq \text { ' 'sci- } f \text { ' }\right) .
\end{aligned}
$$

Notice that the superpreference computed via Thm. 1 is by itself order-preserving. That is, if the profile preference $>_{C}$ is an SPO (WO, TO resp.), the superpreference $>^{+}$computed via Thm. 1 is also an SPO (WO, TO resp.). However, the integration of any domain knowledge might change the order properties of $>^{+}$. For example, in Ex. 6, the profile preference $>_{C}$ is a WO while the superpreference $>^{+}$is not. It is due to the fact that we integrate the domain knowledge price $>0$ in $>^{+}$. Also notice that two important classes of preferences, skyline [1] and p-skyline [9], can be expressed with DNF formulas of the form used in Eqn. (2).

\section{M-relation}

Superpreference is a pruning technique used to filter out tuples not contributing to the best $k$-subsets. It reduces the size of the original relation, which leads to fewer candidate $k$-subsets. In other words, it prunes the inferior $k$-subsets even before the $k$-subset generation. In addition, we often observe another type of redundant candidate $k$-subsets during generation, as shown in Ex. 7. For example, if we have already generated the 3 -subset $\left\{a_{1}, a_{2}, a_{7}\right\}$, we do not need to generate $\left\{a_{1}, a_{2}, a_{9}\right\}$ or $\left\{a_{1}, a_{2}, a_{10}\right\}$ as neither leads to a new profile given the set preference. It is therefore more efficient 
to consolidate $a_{7}, a_{9}, a_{10}$ into a meta-tuple, i.e., an $M$-tuple, $m_{7,9,10}$, and consider only the M-tuple in the generation of candidate $k$-subsets.

Example 7: Add two more tuples to Book in Ex. 1

\begin{tabular}{c|l|c|c|c}
\hline title & genre & rating & price & vendor \\
\hline$a_{1}$ & sci-fi & 5.0 & $\$ 15.00$ & Amazon \\
$a_{2}$ & biography & 4.8 & $\$ 20.00$ & $\mathrm{~B} \& \mathrm{~N}$ \\
$a_{3}$ & sci-fi & 4.5 & $\$ 25.00$ & Amazon \\
$a_{4}$ & romance & 4.4 & $\$ 10.00$ & $\mathrm{~B} \& \mathrm{~N}$ \\
$a_{5}$ & sci-fi & 4.3 & $\$ 15.00$ & Amazon \\
$a_{6}$ & romance & 4.2 & $\$ 12.00$ & $\mathrm{~B} \& \mathrm{~N}$ \\
$a_{7}$ & biography & 4.0 & $\$ 18.00$ & Amazon \\
$a_{8}$ & sci-fi & 3.5 & $\$ 18.00$ & Amazon \\
$a_{9}$ & romance & 4.0 & $\$ 20.00$ & Amazon \\
$a_{10}$ & history & 4.0 & $\$ 19.00$ & Amazon \\
\hline
\end{tabular}

and use the same set preference $\gg_{(\Gamma, C)}$ as that in Ex. 6 . The tuple $a_{7}$ and $a_{9}$ are exchangeable with regard to the set preference, because for every 2 -subset $s$ of Book $2 \backslash\left\{a_{7}, a_{9}\right\}$, extending $s$ with $a_{7}$ or $a_{9}$ leads to the same profile in the profile relation, i.e., $\operatorname{profile}_{\Gamma}\left(s \cup\left\{a_{7}\right\}\right)=\operatorname{profile}_{\Gamma}\left(s \cup\left\{a_{9}\right\}\right)$. By the same argument, $a_{7}, a_{9}$ and $a_{10}$ are mutually exchangeable.

Based on the above idea, we can define an exchangeability relation among tuples. First, we classify the features in a profile schema into three categories, based on their additivity and their appearance in the definition of the set preference. Without loss of generality, the profile schema in a set preference $\gg_{(\Gamma, C)}$ is

$\Gamma=\{\overbrace{\underbrace{\mathcal{F}_{1}, \ldots, \mathcal{F}_{m_{a}}}_{\text {additive }}, \mathcal{F}_{m_{a}+1}, \ldots, \mathcal{F}_{m_{b}}}^{\text {relevant }}, \mathcal{F}_{m_{b}+1} \ldots, \mathcal{F}_{m}\}$, where: (1) The features $\mathcal{F}_{1}, \ldots, \mathcal{F}_{m_{b}}$ are the relevant features appearing in the profile preference formula $C$, denoted by $\Gamma_{\Delta}$, while the features $\mathcal{F}_{m_{b}+1}, \ldots, \mathcal{F}_{m}$ do not appear in $C$; (2) The features $\mathcal{F}_{1}, \ldots, \mathcal{F}_{m_{a}}$ are additive (c.f., Def. 12), while $\mathcal{F}_{m_{a}+1}, \ldots, \mathcal{F}_{m_{b}}$ are not.

Definition 13: Exchangeability Relation. Given a relation $r$, a positive integer $k<|r|$ and a set preference relation $\gg_{(\Gamma, C)}$, an equivalence relation $\approx_{(\Gamma, C)}$ is an exchangeability relation over $r$ if

$$
\begin{aligned}
& t_{1} \approx_{(\Gamma, C)} t_{2} \Rightarrow \\
& t_{1} \in r \wedge t_{2} \in r \wedge\left[\forall s^{\prime} \in(k-1) \text {-subsets }\left(r \backslash\left\{t_{1}, t_{2}\right\}\right),\right. \\
& \text { profile } \left._{\Gamma_{\Delta}}\left(s^{\prime} \cup\left\{t_{1}\right\}\right)=\text { profile }_{\Gamma_{\Delta}}\left(s^{\prime} \cup\left\{t_{2}\right\}\right)\right]
\end{aligned}
$$

in which case, we say that tuple $t_{1}$ and $t_{2}$ are exchangeable.

Given a set preference, there can be more than one exchangeability relation according to Def. 13. For example, the equality relation where each equivalence class contains exactly one tuple is a trivial exchangeability relation. In fact, any equivalence relation contained in an exchangeability relation is another exchangeability relation. However, Prop. 5.3 guarantees a unique optimal exchangeability relation.

Proposition 5.3: Given a relation $r$, a positive integer $k<$ $|r|$ and a set preference relation $\gg_{(\Gamma, C)}$, the optimal exchangeability relation, which contains every possible exchangeability relation over $r$ under $k$ and $\gg_{(\Gamma, C)}$, exists and is unique.

Proof: (Sketch) Denote by $P$ the set of all possible equivalence relations on $r$. It is well known that $P$, when ordered by set containment $(\subseteq)$, form a complete lattice $(P, \subseteq)$, where any subset of $P$ has a supremum. Since all exchangeability relations form a subset of $P$, they have a unique supremum. We can further show that the supremum of any two exchangeability relations is still an exchangeability relation. The supremum of all exchangeability relation is therefore the optimal one.

It is easy to see that the optimal exchangeability relation $\approx_{(\Gamma, C)}$ over the relation $r$ is

$$
\begin{aligned}
& t_{1} \approx_{(\Gamma, C)} t_{2} \Leftrightarrow \\
& t_{1} \in r \wedge t_{2} \in r \wedge\left[\forall s^{\prime} \in(k-1) \text {-subsets }\left(r \backslash\left\{t_{1}, t_{2}\right\}\right),\right. \\
& \text { profile } \left._{\Gamma_{\Delta}}\left(s^{\prime} \cup\left\{t_{1}\right\}\right)=\text { profile }_{\Gamma_{\Delta}}\left(s^{\prime} \cup\left\{t_{2}\right\}\right)\right]
\end{aligned}
$$

Eqn. (4) differs from Eqn. (3) in that it requires a sufficient condition as well.

Example 8: Assume the same set preference $\gg_{(\Gamma, C)}$ in Book 2 of Ex. 6. By Def. 13, one exchangeability relation and its partition are $\approx_{0}=\left\{\left(a_{i}, a_{i}\right) \mid i=1, \ldots, 10\right\}, P_{0}=$ $\left\{\left\{a_{i}\right\} \mid i=1, \ldots, 10\right\}$. Another exchangeability relation and its partition are $\approx_{1}=\left\{\left(a_{i}, a_{i}\right) \mid i=1, \ldots, 10\right\} \cup\left\{\left(a_{7}, a_{9}\right)\right.$, $\left.\left(a_{9}, a_{7}\right),\left(a_{7}, a_{10}\right),\left(a_{10}, a_{7}\right),\left(a_{9}, a_{10}\right),\left(a_{10}, a_{9}\right)\right\}, P_{1}=$ $\left\{\left\{a_{i}\right\} \mid i=1, \ldots, 6,8\right\} \cup\left\{\left\{a_{7}, a_{9}, a_{10}\right\}\right\}$. The exchangeability relation $\approx_{0}$ is the equality relation. Since $P_{0}$ is a refinement of $P_{1}$, i.e., $P_{0} \subset P_{1}$, the exchangeability relation $\approx_{0}$ is not optimal. It is easy to verify that $\approx_{1}$ is optimal since the merge of any equivalence classes in partition $P_{1}$ does not lead to an exchangeability relation over Book2.

We introduce now a profile consolidation optimization using $M$-relations. Given an exchangeability relation $\approx_{(\Gamma, C)}$, an Mrelation contains $M$-tuples, and there is a one-to-one mapping between its $M$-tuples and the equivalence classes of $\approx_{(\Gamma, C)}$. There are various ways to define an M-relation corresponding to an exchangeability relation. In Def. 14 , we show how to define in SQL an M-relation corresponding, under certain conditions, to the optimal exchangeability relation.

In Def. 14, the M-relation is defined using a query on the distinct values of attributes corresponding to the relevant features $\mathcal{F}_{1}, \ldots, \mathcal{F}_{m_{b}}$. Moreover, for the additive relevant features $\mathcal{F}_{1}, \ldots, \mathcal{F}_{m_{a}}$, tuples are grouped by their contribution to the values of the additive features over sets: $f_{i}$ is the base of the additive feature $\mathcal{F}_{i}$. The M-relation also keeps track of the number of tuples consolidated into an M-tuple, using the special attribute $A_{\text {cnt }}$.

Definition 14: M-relation using SQL. Given a relation $r$ with a schema $R$, a non-negative integer $k$ and a set preference $\gg_{(\Gamma, C)}$, define the M-relation schema $O=\left\langle A_{1}, \ldots, A_{m_{a}}\right.$, $\left.A_{m_{a}+1}, \ldots, A_{m_{c}}, A_{c n t}\right\rangle$, and the M-relation $o$ by the following SQL query:

SELECT $f_{1}(\mathrm{R})$ AS $A_{1}, \ldots, f_{m_{a}}(\mathrm{R})$ AS $A_{m_{a}}$, $\operatorname{attrs}\left(\mathcal{F}_{m_{a}+1}, \ldots, \mathcal{F}_{m_{b}}\right)$, count (*) AS $A_{\text {cnt }}$

FROM R GROUP BY $A_{1}, \ldots, A_{m_{a}}, \operatorname{attrs}\left(\mathcal{F}_{m_{a}+1}, \ldots, \mathcal{F}_{m_{b}}\right)$ where: (1) $\mathrm{R}$ is a tuple range variable. (2) $f_{i}(\mathrm{R})$, $i=1, \ldots, m_{a}$ is the base of the additive feature $\mathcal{F}_{i}$. (3) $\operatorname{attrs}\left(\mathcal{F}_{m_{a}+1}, \ldots, \mathcal{F}_{m_{b}}\right)$ is the set of attributes mentioned in the definitions of the features $\mathcal{F}_{m_{a}+1}, \ldots, \mathcal{F}_{m_{b}}$, say $\left\{A_{m_{a}+1}, \ldots, A_{m_{c}}\right\}$. (4) $A_{c n t}$ is a special attribute in the 
M-relation schema tracking the number of tuples consolidated into a single M-tuple in the M-relation.

Example 9: In Ex. 7, the M-relation schema is $\left\langle A_{5}, A_{6}, A_{c n t}\right\rangle$, which is determined by the set preference. In order to illustrate the case where there are non-additive relevant features, and more importantly the concept of projection to relevant features, we assume the non-additive feature $\mathcal{F}_{3}$ is also a relevant feature in the set preference. Thus, the M-relation schema becomes $\left\langle A_{5}, A_{6}, A_{3}, A_{c n t}\right\rangle$, and the M-relation is generated via the following SQL query: SELECT

CASE WHEN r.genre='sci-fi' THEN r.price

ELSE 0 END AS $A_{5}$, r.rating AS $A_{6}$,

r.vendor AS $A_{3}$, count (*) AS $A_{\text {cnt }}$

FROM Book $2 r$ GROUP BY $A_{5}, A_{6}, A_{3}$

In the following M-relation $o^{\prime}$, the subscripts of each M-tuple are the indices of the tuples consolidated into it.

\begin{tabular}{l|l|l|l|l}
\hline & $A_{5}$ & $A_{6}$ & $A_{3}$ & $A_{\text {cnt }}$ \\
\hline$m_{1}$ & $\$ 15.00$ & 5.0 & Amazon & 1 \\
$m_{2}$ & $\$ 0.00$ & 4.8 & B\&N & 1 \\
$m_{3}$ & $\$ 25.00$ & 4.5 & Amazon & 1 \\
$m_{4}$ & $\$ 0.00$ & 4.4 & B\&N & 1 \\
$m_{5}$ & $\$ 15.00$ & 4.3 & Amazon & 1 \\
$m_{6}$ & $\$ 2.00$ & 4.2 & B\&N & 1 \\
$m_{7,9,10}$ & $\$ 0.00$ & 4.0 & Amazon & 3 \\
$m_{8}$ & $\$ 18.00$ & 3.5 & Amazon & 1 \\
\hline
\end{tabular}

Notice that the actual M-relation $O$ in Ex. 7 is the above $o^{\prime}$ relation with the columns $A_{5}, A_{6}, A_{\text {cnt }}$ only.

In Ex. $9, f_{5}$, the base of the additive feature $\mathcal{F}_{5}$, is expressed via a CASE statement. In the CASE statement, the ELSE statement gives a default value to the new feature if the WHEN condition is not satisfied. The default value is 0 for $A_{5}$ in Ex. 9. In general, it can be any constant. M-relations identify the exchangeable tuples in Ex. 7.

Theorem 2: For a DNF profile preference formula $C$ in the form of Eqn. (2), a relation $r$ and $k<|r|$, if all relevant features are additive, then the exchangeability relation corresponding to the M-relation in Def. 14 is optimal over $r$.

Example 10: In Ex. 9, the partition generated by the M-relation defined in Def. 14 is $\left\{\left\{a_{1}\right\}, \ldots,\left\{a_{6}\right\}\right.$, $\left.\left\{a_{7}, a_{9}, a_{10}\right\},\left\{a_{8}\right\}\right\}$, which is the exact partition corresponding to the optimal exchangeability relation defined by Eqn. (4).

\section{Computing Profiles via M-relations}

Our goal is to directly compute the profiles from an Mrelation. The subtlety lies in that if we compute profiles only from the $k$-subsets of the M-relation, we might miss some of the profiles in the original relation. Say $k=2$, and an M-tuple $m_{1,2}$ corresponds to tuples $t_{1}$ and $t_{2}$ in the original relation $r$. We have a profile computed from the 2 -subset $\left\{t_{1}, t_{2}\right\}$ in the profile relation $\gamma$. However, we cannot compute this profile from a $k$-subset of the M-relation, as any $k$-subset contains at most one M-tuple $m_{1,2}$ and $\left\{m_{1,2}, m_{1,2}\right\}$ is not a 2 -subset of the M-relation. Hence, in order to compute the exact profiles of the original relation, we need to compute profiles from the $k$-multisubsets of an M-relation.

Definition 15: $k$-multisubset. Given an M-relation $o$ and a positive integer $k, k<\sum_{m_{i} \in o} m_{i}$. $A_{c n t}$, a $k$-multisubset $s$ of $o$ is a multiset of $o$ with cardinality $k$, and the number of occurrences of each M-tuple does not exceed its $A_{c n t}$ value. Denote by $k$-multisubsets $(o)$ the set of all $k$-multisubsets of $o$.

Note that Def. 15 is the multisubset version of Def. 9. We can easily extend Def. 3-12 to their multisubset counterparts (details omitted).

Theorem 3: Assume $o$ is an M-relation corresponding to a relation $r$. For each $s \in k$-subsets $(r)$, there is a $s^{\prime} \in$ $k$-multisubsets $(o)$, such that profile $\Gamma_{\Gamma_{\Delta}}(s)=$ profile $_{\Gamma_{\Delta}}\left(s^{\prime}\right)$, and vice versa.

Thm. 3 guarantees that the projection of the original profile relation to relevant features computed from the M-relation is an onto function. Therefore, we can compute the projection by evaluating those features of $k$-multisubsets. Alg. 4 uses M-relations to compute the best $k$-subsets. Alg. 4 differs from $\mathrm{Alg} .1$ in the use of an M-relation and a $k$-multisubset generator. The number of occurrences of an M-tuple in a candidate $k$-multisubset is bounded from above by the number of tuples consolidated into it. It is therefore crucial to the $k$ multisubset generation that the M-relation records this number for each M-tuple.

\section{Algorithm 4 (MREL) M-relation Algorithm}

Require: a profile schema $\Gamma$, an SPO profile preference relation $>_{C}$, a relation $r$ and a positive integer $k, k<|r|$

Ensure: the best $k$-subsets of $r$ under the set pref. $\gg_{(\Gamma, C)}$

1: Compute the M-relation of $r$ based on $\Gamma$ and $>_{C}$.

2: Generate all $k$-multisubsets of $o$ and for each compute the profile features in $\Gamma$ relevant to $C$, i.e., in $\Gamma_{\Delta}$, to obtain $\gamma^{\prime}$, i.e. the projection of the profile relation $\gamma$ onto $\Gamma_{\Delta}$.

3: Compute $\gamma^{\prime \prime}=\omega_{C}\left(\gamma^{\prime}\right)$ using any winnow evaluation algorithm.

4: Retrieve the subsets whose profile projections correspond to the elements of $\gamma^{\prime \prime}$.

The profile of a $k$-multisubset in an M-relation is the projection of the profile of the corresponding $k$-subset(s) in the original relation to relevant features. Notice that the M-relation contains all the information needed to compute the projection of this profile. For an additive relevant feature, we keep each M-tuple's contribution to the value of this feature. For a nonadditive relevant feature, we keep the values of every attribute involved in the evaluation of this feature. Therefore, in order to compute the projection of this profile, we simply add up each M-tuple's contribution for additive relevant features, and compute the values of non-additive relevant features as we would do for a $k$-subset.

Example 11: Continuing Ex. 9, the following table gives a few examples of candidate $k$-subsets $(k=3)$ in Book2 and their corresponding $k$-multisubsets in the M-relation $o$, together with their profiles in the profile relation $\gamma$.

\begin{tabular}{c|l|l}
\hline \hline$k$-subsets of Book2 & $k$-multisubsets of $o$ & profile in $\gamma$ \\
\hline \hline$\left\{a_{1}, a_{2}, a_{7}\right\}$, & & \\
$\left\{a_{1}, a_{2}, a_{9}\right\}$, & $\left\{m_{1}, m_{2}, m_{7,9,10}\right\}$ & $(\$ 15.00,13.8)$ \\
$\left\{a_{1}, a_{2}, a_{10}\right\}$ & & \\
\hline$\left\{a_{1}, a_{7}, a_{9}\right\}$, & & \\
$\left\{a_{1}, a_{7}, a_{10}\right\}$, & $\left\{m_{1}, m_{7,9,10}, m_{7,9,10}\right\}$ & $(\$ 15.00,13.0)$ \\
$\left\{a_{1}, a_{9}, a_{10}\right\}$ & & \\
\hline
\end{tabular}


In fact, a $k$-subset generator of Book 2 will enumerate all $\left(\begin{array}{c}10 \\ 3\end{array}\right)=120$ candidate $k$-subsets, while a $k$-multisubset generator of the M-relation will only enumerate $\left(\begin{array}{l}7 \\ 3\end{array}\right)+\left(\begin{array}{l}7 \\ 2\end{array}\right)+$ $\left(\begin{array}{l}7 \\ 1\end{array}\right)+\left(\begin{array}{l}7 \\ 0\end{array}\right)=64 k$-multisubsets, where $\left(\begin{array}{l}7 \\ i\end{array}\right)$ stands for the case where the multisubset contains $3-i m_{7,9,10}$, and $i$ non- $m_{7,9,10}$ M-tuple(s). Since the feature $\mathcal{F}_{5}$ and $\mathcal{F}_{6}$ are both additive, the evaluation of $\mathcal{F}_{5}$ changes to SELECT sum $\left(A_{5}\right)$ FROM $\$ S$ and the evaluation of the feature $\mathcal{F}_{6}$ changes to $\operatorname{SELECT}$ sum $\left(A_{6}\right)$ FROM $\$ \mathrm{~S}$. We can compute a profile from a $k$-multisubset by computing its features. For example, $s=\left\{m_{1}, m_{2}, m_{7,9,10}\right\}$, then $\mathcal{F}_{5}(s)=$ $m_{1} \cdot A_{5}+m_{2} \cdot A_{5}+m_{7,9,10} \cdot A_{5}=\$ 15.00+\$ 0.00+\$ 0.00=$ $\$ 15.00$, and $\mathcal{F}_{6}(s)=m_{1} \cdot A_{6}+m_{2} \cdot A_{6}+m_{7,9,10} \cdot A_{6}=5.0+$ $4.8+4.0=13.8$. The profile of the multiset $s$ is $(\$ 15.00,13.8)$. The profile relation $\Gamma$ contains 64 distinct profiles.

In Ex. 11, the $k$-multisubset generator eliminates all redundancy in the subset generation: each candidate $k$-multisubset returned by the generator leads to a distinct new profile. Though it is not always the case in general, a $k$-multisubset generator still reduces the number of subsets generated significantly in most cases. The benefit of M-relations comes in three folds: (1) Selectivity of attribute values. The Mrelation is defined by a GROUP BY SQL query in Def. 14, which suggests that low selectivity of attribute values will lead to fewer M-tuples. (2) Selectivity of WHERE conditions in feature definitions. Since every feature definition is also a "mini-SQL" query, it is likely that a tuple not satisfying the WHERE condition of the feature definition will contribute a default value in the feature evaluation. In fact, such default values exist for all additive features. Recall that the M-relation definition query in Ex. 9 has default value 0 for attribute $A_{5}$. The default values fully exploit the selectivity of the WHERE condition. A highly selective WHERE condition will lead to more tuples with the default value, which are more likely to be consolidated into one tuple in the M-relation. This in turn suggests a smaller M-relation. (3) Non-relevant attributes. The projection to attributes relevant to the set preference reduces the redundancy in $k$-subset generation.

\section{E. Hybrid Approaches}

Superpreference and $M$-relation target two different aspects of pruning: superpreference filters out tuples which cannot be a part of any best $k$-subset, while $M$-relation consolidates exchangeable tuples and processes $k$-subsets in groups. It is natural to combine them to achieve synergistic pruning. We propose here two possible combinations of those two optimizations: SM and MS. Without loss of generality, assume we are given a relation $r$, a positive integer $k<|r|$ and a set preference $\gg_{(\Gamma, C)}$.

(SM) Superpreference followed by M-relation. This algorithm is rather straightforward. We use the technique in superpreference to filter out tuples with cover size at least $k$, and then apply $M$-relation to the reduced input to compute the final results. The exact algorithm is illustrated in Alg. 5.

(MS) M-relation followed by Superpreference. In this algorithm (Alg. 6), we apply the superpreference technique to the M-relation. We compute the M-relation as before. In order to apply the superpreference optimization to an M-relation, we need to generalize the notions of superpreference and cover to M-relations. Recall that, in Def. 11, a tuple $t_{1}$ is superpreferred to a tuple $t_{2}$ iff it is more beneficial to extend every (k-1)-subset with $t_{1}$ instead of $t_{2}$ to get a $k$-subset. The superpreference relation $>_{o}^{+}$in an M-relation $o$ is based on the same idea: an M-tuple $m_{1}$ is superpreferred to an M-tuple $m_{2}$ iff it is more beneficial to extend every (k-1)-multisubset with $m_{1}$ instead of $m_{2}$ to get a $k$-multisubset. Similarly, the cover of an M-tuple $m$ is the multiset of M-tuples, where each M-tuple $m^{\prime}$ dominates $m$ under $>_{o}^{+}$, and has exactly $m^{\prime}$. $A_{\text {cnt }}$ occurrences in the cover, i.e., cover $(m)=$ $\left\{m^{\prime}, m^{\prime}\right.$ repeats $m^{\prime} . A_{c n t}$ times $\left.\mid m^{\prime} \in o, m^{\prime}>_{o}^{+} m\right\}$.

Algorithm 5 (SM) Super-MRel Hybrid Algorithm

Require: a profile schema $\Gamma$, an SPO profile preference relation $>_{C}$, a relation $r$, a positive integer $k, k<|r|$ and $>^{+}$corresponding to $C^{+}$

Ensure: the best $k$-subsets of $r$ under the set pref. $\gg_{(\Gamma, C)}$

1: Do pairwise comparison between tuples in $r$ based on $>^{+}$, and determine cover $(t)$ for each $t \in r$.

2: Let $r^{\prime}=\{t \in r||$ cover $(t) \mid<k\}$.

3: Return the result of the M-relation Algorithm (Alg. 4) with the input relation $r^{\prime}$ and the profile preference relation $>_{C}$ restricted to $r^{\prime}$ instead.

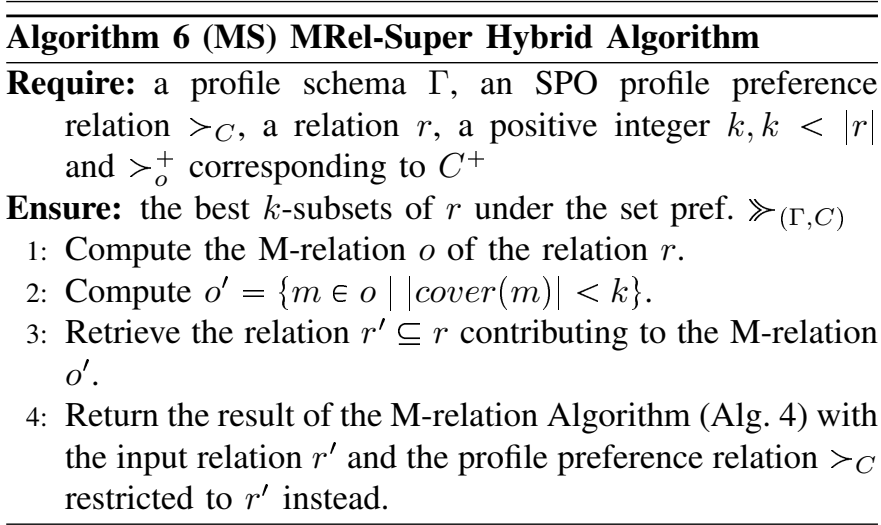

\section{EXPERIMENTS}

We report here an experimental study of the best-subset generation algorithms proposed. We implemented all algorithms in $\mathrm{C}++$ and ran experiments on a machine with Intel Core2 1.66GHz CPU running Cygwin on Windows XP with 2GB memory. We used a real dataset containing the information of 8000 book quotes from Amazon. The data schema is $\langle$ title, genre, rating, price, vendor $\rangle$. We implemented five algorithms: NAIVE, SUPER, MREL, SM, MS. NAIVE is the basic algorithm in Alg. 1. SUPER and MREL are the implementation of Alg. 2 and Alg. 4, respectively. SM and MS are the hybrid algorithms in Sec. V-E.

The running time of each algorithm is composed of three major operations: (1) Preprocessing: superpreference filtering and/or the M-relation generation; (2) Generation: candidate $k$-subset (or $k$-multisubset) generation; (3) Winnow. Denote by $g$ the number of sets generated in Generation. $g$ is the 
determinant factor of the running time. It is a direct indicator of the Generation step and also the input of the Winnow step, which is an operation quadratic of its input size $g$. Our experiments demonstrate a strong correlation between $g$ and the running time. In the set of experiments in Sec. VI-B, the correlation is $94.6 \%$. Therefore, we focus on $g$ for measuring the performance. Our experiments are practical to handle up to 8000 tuples. Further optimizations are needed for larger datasets. Recall the definitions of feature $\mathcal{F}_{5}$ and $\mathcal{F}_{6}$ in Ex. 6 . Furthermore, we define the following features:

$$
\begin{aligned}
& \mathcal{F}_{9} \equiv \text { SELECT sum(rating) FROM } \$ \text { S } \\
& \text { WHERE genre='sci-fi' } \\
& \mathcal{F}_{10} \equiv \text { SELECT sum(price) FROM } \$ S \\
& \mathcal{F}_{11} \equiv \text { SELECT count (title) FROM } \$ S \\
& \mathcal{F}_{12} \equiv \text { SELECT Sum(rating) } \mathrm{FROM} \$ \mathrm{~S} \\
& \text { WHERE rating }>=4.0
\end{aligned}
$$

The set preferences used in the experiments are listed in Table I. The corresponding superpreferences and M-relation generation SQL queries are listed in Table II, respectively. Notice that the only difference between SP1 and SP2 is that in SP1 we apply the WHERE condition in $\mathcal{F}_{5}$ which aggregates on price, while in SP2 we apply it to $\mathcal{F}_{9}$ which aggregates on rating. We intend to see the influence of the selectivity of attributes. In SP1, the attribue in $\mathcal{F}_{5}$, i.e., price, affects less tuples due to the WHERE condition, therefore the selectivity of the attribute in $\mathcal{F}_{6}$, i.e., rating, is dominating. Similarly, in SP2, the selectivity of price is dominating. Note that SP1 has been used extensively in our running examples.

\begin{tabular}{|l|l|l|}
\hline Pref. & $\begin{array}{l}\text { Prof. } \\
\text { Schema } \Gamma\end{array}$ & Profile Pref. Formula C \\
\hline SP1 & $\left\langle\mathcal{F}_{5}, \mathcal{F}_{6}\right\rangle$ & $\mathcal{F}_{5}\left(s_{1}\right)<\mathcal{F}_{5}\left(s_{2}\right) \wedge \mathcal{F}_{6}\left(s_{1}\right)>\mathcal{F}_{6}\left(s_{2}\right)$ \\
SP2 & $\left\langle\mathcal{F}_{9}, \mathcal{F}_{10}\right\rangle$ & $\mathcal{F}_{9}\left(s_{1}\right)>\mathcal{F}_{9}\left(s_{2}\right) \wedge \mathcal{F}_{10}\left(s_{1}\right)<\mathcal{F}_{10}\left(s_{2}\right)$ \\
SP3 & $\left\langle\mathcal{F}_{11}, \mathcal{F}_{12}\right\rangle$ & $\mathcal{F}_{11}\left(s_{1}\right)>\mathcal{F}_{11}\left(s_{2}\right) \wedge \mathcal{F}_{12}\left(s_{1}\right)>\mathcal{F}_{12}\left(s_{2}\right)$ \\
\hline
\end{tabular}

TABLE I

SET PREFERENCES

Summary of Experiments. We draw the following conclusions from the experimental results detailed below:

- SUPER, MREL, SM and MS are all effective in reducing the number of sets generated in Generation;

- The relative efficiency of the four optimization algorithms depends on the set preference in question, in particular, the selectivity of the attributes and the WHERE conditions in the feature definitions. In general, low attribute selectivity and high WHERE condition selectivity enhance the performance. The best algorithm for each set preference generates only $0.01 \%$ of the candidate $k$-subsets generated by NAIVE.

- The best of SUPER, MREL, SM and MS for each set preference also improve the scalability with input size $n$ and $k$ by several orders of magnitude.

- The hybrid algorithms SM and MS outperform the standalone optimizations SUPER and MREL.

\section{A. Performance}

In our first experiment, we wanted to study how much computation we could save by applying superpreference and/ or M-relation. Let $k=3$. We tested the dataset up to 1000 tuples as some algorithms do not scale up well with $n$.

Fig. 1(a), 1(b) and 1(c) illustrate the increase of the number of sets produced by Generation $(g)$ with the increase of input size $n$ for SP1, SP2 and SP3, respectively. For the six datasets of different sizes used, Fig. 1(d), 1(e) and 1(f) show the average ratio of the number of sets generated in each algorithm to that in NAIVE using the logarithmic $y$-scale.

As we can see from Fig. 1, for standalone optimization techniques SUPER and MREL, the relative efficiency depends on the set preference. MREL is more efficient in SP1 and SP3 (Fig. 1(d), 1(f)), while SUPER is more efficient in SP2 (Fig. 1(e)). If we compare SP1 and SP2 in juxtaposition, it is clear the different reactions to optimizations is caused by the different selectivity of the attributes rating and price: in our largest dataset, there are 9 distinct rating values and 406 distinct price values. For SUPER, the differences between the superpreferences of SP1 and SP2 are underlined. $\left(t_{1}\right.$. rating $>t_{2}$. rating $\wedge t_{2}$. genre $=$ 'sci- $f$ '

$>^{+}$in $\mathrm{SP} 1 \equiv \wedge t_{1}$. price $<t_{2}$. price $) \vee\left(\underline{t_{1} . \text { rating }>t_{2} . \text { rating }}\right.$ $\wedge t_{2}$. genre $=$ 'sci-fi' $\wedge t_{1}$.genre $\neq$ 'sci-fi') $\left(t_{1}\right.$. rating $>t_{2}$. rating $\wedge t_{1}$.genre $=$ 'sci- $f$ '

$>^{+}$in SP2 $\equiv \wedge t_{1}$.price $<t_{2}$.price $) \vee\left(t_{1}\right.$.price $<t_{2}$. price $\wedge t_{1}$. genre $=$ 'sci-fi' $\wedge t_{2}$. genre $\neq$ 'sci-fi')

SUPER is more effective in SP2 because the selectivity of price is higher than that of rating and more tuples are superpreferred in SP2. For MREL, recall that the selectivity of rating dominates in SP1, while the selectivity of price dominates in SP2. The selectivity of rating is much lower than that of price. We are thus able to consolidate on average more tuples into one M-tuple in SP1. Therefore, MREL is more effective in SP1. For SP3, the WHERE conditions in both features of SP3 are more restricted than that in SP1, i.e., the condition in $\mathcal{F}_{11}$ requires price $<20.00$, in addition to the condition genre=' $\mathrm{SCi}-\mathrm{fi}^{\prime}$ used in $\mathcal{F}_{5}$. MREL is able to benefit more from low selectivity and therefore achieves higher efficiency in SP3.

The hybrid algorithms SM and MS benefit from both superpreferences and M-relations, and in general have better performance than SUPER and MREL. An interesting phenomenon is that SM outperforms MS when MREL outperforms SUPER (c.f., Fig. 1(d), 1(f)), and MS outperforms SM when SUPER outperforms MREL (c.f., Fig. 1(e)). For all three set preferences (SP1, SP2 and SP3), the most efficient algorithm generates only about $0.01 \%$ of the candidate $k$ subsets generated by NAIVE.

Not only do the optimizations reduce the number of sets generated in Generation, but also improve the scalability with the input size $n$. In all three cases, i.e., SP1-3, (Fig. 1(a), 1(b) and $1(\mathrm{c})$ ), the best algorithm displays a much slower growth compared to that of NAIVE.

Fig. 2 illustrates the increase of $g$ with the increase of $k$ when $n=100$. By our theoretical analysis, increasing $k$ by 1 raises the complexity of NAIVE from $O\left(n^{k}\right)$ to $O\left(n^{k+1}\right)$. It is not surprising that $g$ values are off the chart when $k>4$ for NAIVE. The performances of the other four algorithms again depend on the specific set preference. The best algorithm 


\begin{tabular}{|c|c|c|}
\hline Pref. & Superpreference & M-relation Generating SQL \\
\hline SP1 & $\begin{array}{l}t_{1} . \text { rating }>t_{2} . \text { rating } \wedge t_{2} . \text { genre }=\text { 'sci-fi' } \\
\wedge\left(t_{1} . \text { price }<t_{2} . \text { price } \vee t_{1} . \text { genre } \neq \text { 'sci-fi' }\right)\end{array}$ & $\begin{array}{l}\text { SELECT *, count }(*) \text { AS } A_{\text {cnt }} \text { FROM } \\
\text { (SELECT CASE WHEN r.genre='sci-fi' THEN r.price } \\
\text { ELSE } 0 \text { END AS } A_{5}, \text { r.rating AS } A_{6} \text { FROM r) } \\
\text { GROUP BY } A_{5}, A_{6}\end{array}$ \\
\hline SP2 & $\begin{array}{l}t_{1} \cdot \text { price }<t_{2} . \text { price } \wedge t_{1} . \text { genre }=\text { 'sci-fi' } \\
\wedge\left(t_{1} \cdot \text { rating }>t_{2} . \text { rating } \vee t_{2} . \text { genre } \neq \text { 'sci-f }{ }^{\prime}\right)\end{array}$ & $\begin{array}{l}\text { SELECT *, count }(*) \text { AS } A_{c n t} \text { FROM } \\
\quad(\text { SELECT CASE WHEN r.genre=' sci-fi' THEN r.rating } \\
\text { ELSE } 0 \text { END AS } A_{9}, r \cdot \text { price AS } A_{10} \text { FROM r) } \\
\text { GROUP BY } A_{9}, A_{10}\end{array}$ \\
\hline SP3 & $\begin{array}{l}t_{1} . \text { genre }=\text { 'sci-fi' } \wedge t_{1} . \text { price }<20.00 \\
\wedge\left(t_{2} . \text { genre } \neq \text { 'sci-fi' } \vee t_{2} . \text { price } \geqslant 20.00\right) \\
\wedge t_{1} . \text { rating } \geqslant 4.0 \\
\wedge\left(t_{2} . \text { rating } \leqslant 4.0 \vee t_{1} . \text { rating }>t_{2} . \text { rating }\right)\end{array}$ & $\begin{array}{l}\text { SELECT } *, \text { count }(*) \text { AS } A_{\text {cnt }} \text { FROM } \\
\text { (SELECT CASE WHEN r.genre='sci-fi' and r.price }<20.00 \\
\text { THEN } 1 \text { ELSE } 0 \text { END AS } A_{11}, \\
\text { CASE WHEN r.rating } \geqslant 4.0 \text { THEN r.rating } \\
\text { ELSE } 0 \text { END AS } A_{12} \\
\text { FROM r) } \\
\text { GROUP BY } A_{11}, A_{12}\end{array}$ \\
\hline
\end{tabular}

TABLE II

SUPERPREFERENCES AND M-RELATION CONSTRUCTION IN SQL

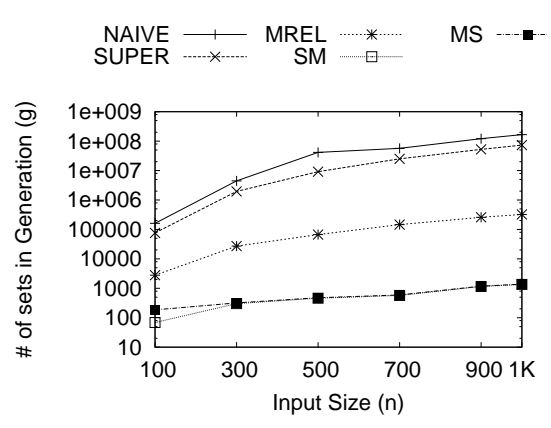

(a) \# of sets v.s. $n$ (SP1)

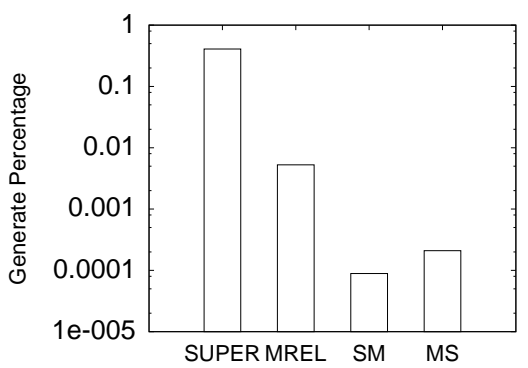

(d) Avg Generate Percentage (SP1)

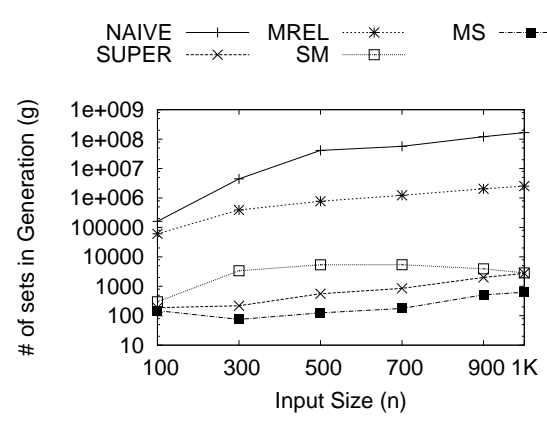

(b) \# of sets v.s. $n$ (SP2)

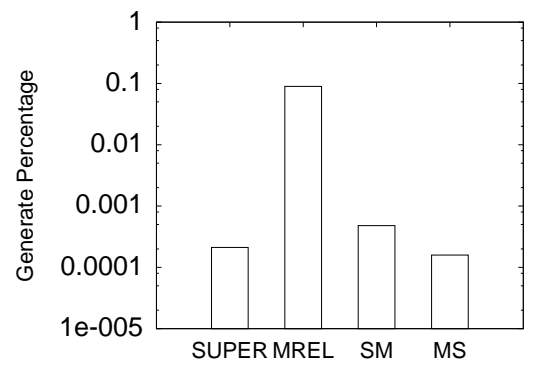

(e) Avg Generate Percentage (SP2)

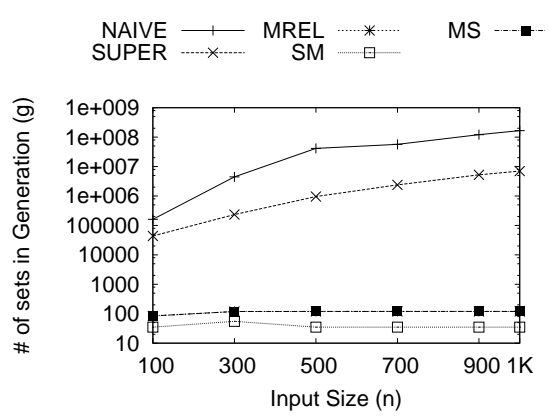

(c) \# of sets v.s. $n$ (SP3)

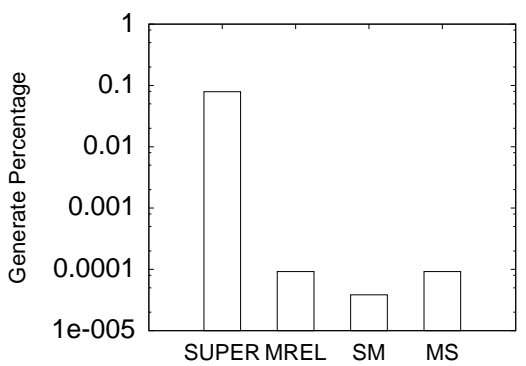

(f) Avg Generate Percentage (SP3)

Fig. 1. Performance of Different Algorithms under Varying Input Size $n$

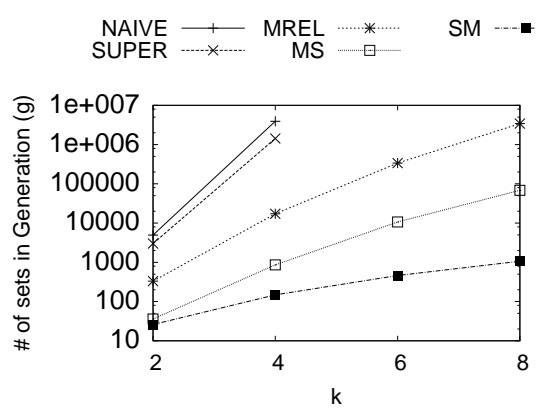

(a) \# of sets v.s. $k$ (SP1)

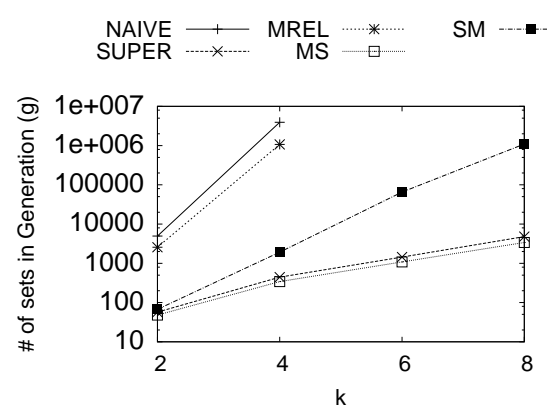

(b) \# of sets v.s. $k$ (SP2)

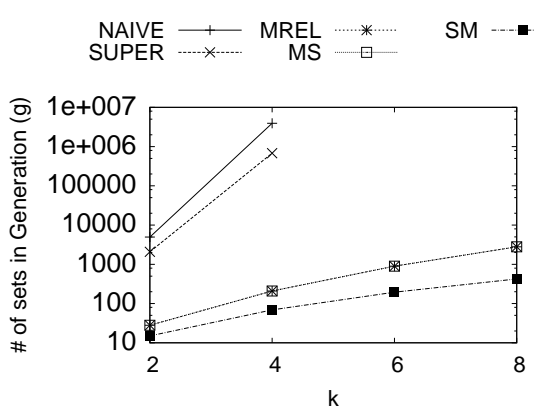

(c) \# of sets v.s. $k$ (SP3)

Fig. 2. Performance of Different Algorithms under Varying $k$ 


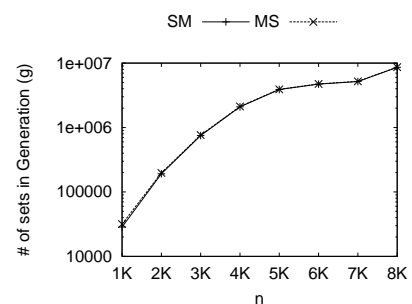

(a) Generate Size ( $g$ )

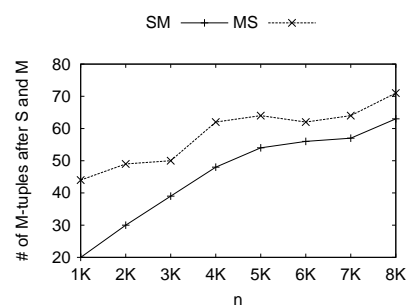

(b) \# of M-tuples after S and M

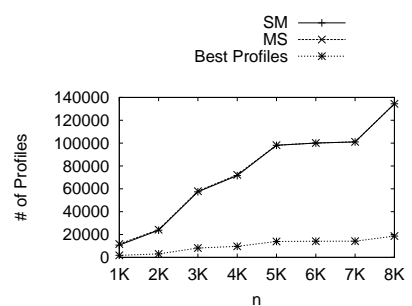

(c) \# of Profiles

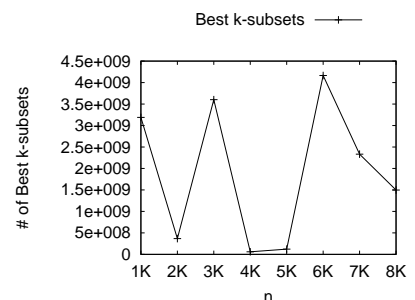

(d) \# of Best $k$-subsets

Fig. 3. SM v.s. MS in SP1

for each set preference shows a between linear and quadratic scale-up with the increase of $k$. In particular, the exponential blowup predicted by the theoretical analysis does not happen for those best-performing algorithms.

It is worth noticing that regardless of which set preference is used, the performance of different algorithms is stable with the increase of $n$ or $k$, as the curves of each algorithms do not cross each other in Fig. 1 or Fig. 2. The figures illustrate that it is crucial to choose the right algorithm given a set preference. A practical strategy would be to take a small sample of data, and try out the algorithms with a small $k$ in order to pick the best. Notice that, even though the best of SM and MS is better than the best of SUPER and MREL, SUPER and MREL are simpler and involve less preprocessing, and thus could be desirable in some cases.

\section{B. $S M$ v.s. $M S$}

In the second set of experiments, we took a closer look at the two best algorithms: SM and MS. In Fig. 1, SP2 and SP3 are biased towards the superpreference optimization and M-relation optimization, respectively. We therefore focused on SP1, which is less biased towards either optimization, when comparing SM and MS.

In Fig. 3, we compare the analytics of the hybrid algorithms SM and MS when $k=5$ and $n$ varies from 1000 to 8000 in SP1. Fig. 3(a) illustrates the number of sets generated in Generation for different parameter settings. Fig. 3(b) shows the number of M-tuples after the superpreference step and the M-relation step in SM and MS. Those are the M-tuples participating in the $k$-multisubset generation in both algorithms. This number has a positive correlation with the number of $k$ multisubsets generated (Fig. 3(a)). In Fig. 3(b), we can see that SM leads to $10 \% \sim 50 \%$ fewer M-tuples compared to those in MS. The saving decreases with the increase of $n$. For each parameter setting in Fig. 3(a), MS generates slightly more sets than SM does. The difference is relatively small compared to $g$. As a result, the two lines in Fig. 3(a) almost overlap with each other. Fig. 3(c) displays the number of profiles computed in SM and MS. The two lines also overlap with each other, since SM generates only slightly fewer profiles than MS does. As a reference, we also illustrate the number of best profiles in the profile relation in Fig. 3(c). This number grows slower than the number of profiles generated in both algorithms, which suggests room for further improvement.
For completeness, we show the result size: the number of best $k$-subsets in each parameter setting in Fig. 3(d). Both SM and MS correctly identify all the best $k$-subsets. The fluctuation in the number of best $k$-subsets can be explained by the existence of super tuples that are superpreferred to many tuples, and therefore lead to fewer best $k$-subsets. However, the number of such super tuples is not related to the size of the input. In general, the number of best $k$-subsets is huge, i.e., in the order of $10^{8} \sim 10^{9}$. In fact, the result size in Fig. 3(d) is $2 \sim 5$ orders of magnitude larger than the number of candidate sets generated (Fig. 3(a)), which illustrates the compactness of the M-relation representation.

\section{RELATED WORK}

There are many papers on preferences over tuples using either a qualitative or a quantitative approach. However, there are only a few works on preferences over sets [6], [7], [8].

Binshtok et al. [8] is conceptually the closest to our work. It addresses the problem of finding an optimal subset of a set of items according to given set preferences. The language for specifying such preferences uses the attribute values of individual items within the set. Each set property is based on the number of items satisfying a certain predicate. It is either an integer value (Class 1), i.e., the number of items satisfying the predicate), or a boolean value (Class 2), i.e., whether the number of items satisfying the predicate is $>k$. Given a collection of set properties, a set preference is specified as either a TCP-net [12] or a scoring function. Binshtok et al. [8] gives heuristic search algorithms for finding an optimal subset. Binshtok et al. [8] considers subsets of any cardinality. For fixed-cardinality subsets, the language in [8] can easily be expressed in our framework with a simple extension of Def. 6 to boolean features. Each Class 1 set property in [8] can be translated to an aggregate subset feature with the count aggregate. Those features are additive.

For simplicity, we do not discuss boolean features in Def. 6. The extension to boolean features can be easily accomplished by introducing a relational operator (or an appropriate userdefined function) in the SQL definition: SELECT expr $\theta$ constant FROM $\$$ S WHERE condition, where $\theta \in\{=$, $\neq,<,>, \leqslant, \geqslant\}$. Each Class 2 set property in [8] can be translated to such a boolean feature. Such boolean features are additive when $\theta \in\{<,>, \leqslant, \geqslant\}$ and its numeric counterpart is additive. Otherwise, they are non-additive. The preference 
model in [8], i.e., either a scoring function or a TCP-net set preference, can be captured by our set preference relation as well. General aggregate features are not supported in [8]. The largest dataset experimented on in [8] is of a comparable size to those used in our experiments.

desJardins et al. [7] focuses on fixed-cardinality set preferences. It considers two subset features: diversity and depth, and the set preference as an objective function of maximizing the linear combination of diversity and depth. Again, those cases can be expressed in our framework. The subset features depth and diversity are weighted sums of the depth of attributes and diversity of attributes, respectively. The depth of an attribute is a utility function of the most desirable attribute values of the set elements, which can be captured by a feature definition with a WHERE clause specifying the desirable condition and a count aggregate ${ }^{1}$ in the SELECT clause. The diversity of an attribute is defined as 1 minus the skew of that attribute's values in the set. The computation of skew requires the values of mean, mode and standard deviation of that attribute. Mean and mode can be captured by aggregates avg and max, respectively. It is well-known that the standard deviation equals the square root of the second moment minus the square of the mean. We can capture the second moment of attribute $A$ by specifying sum $(\mathrm{A} \star \mathrm{A})$ in the SELECT clause. Features derived from depth, and features corresponding to the mean and the second moment of an attribute are additive, while features corresponding to the mode of an attribute are not. The preference model in [7] is maximizing an objective function composed of a linear combination of depth and diversity. This can be easily captured by our set preference relation.

We have just shown that we can express set preferences in both [8] and [7] using our current framework. In order to efficiently evaluate the set preferences derived from [8], [7], the M-relation optimization is always applicable. For the superpreference optimization, if the set preference formula derived can be written as a DNF in Eqn. (2), then we can apply the superpreference optimization in a systematic manner based on Thm. 1. Moreover, the features and the preference model in [7] is of a special form, so that a specialized optimization schema can be designed to achieve better optimization results.

Guha et al. [6] considers a new class of queries called OPAC (optimization and parametric aggregation constraints) queries. Such queries aim at identifying sets of tuples that constitute the solutions of optimization problems. Guha et al. [6] considers subsets of any cardinality. The evaluation of general OPAC queries is NP-hard, and the specific form of the OPAC queries discussed in [6] corresponds to the wellknown multi-attribute knapsack problem and is therefore NPcomplete. Approximation algorithms are given for query evaluation. For fixed-cardinality subsets, again, in our framework the atomic aggregation constraints can be captured by $k$-subset features, and the parameters and the objective function by the preference formula over profiles.

\footnotetext{
${ }^{1}$ In cases where values outside the desirable range are penalized to varying degrees, we can define one feature for each degree of penalization.
}

\section{CONClusions And Future Work}

We demonstrated a "logic + SQL" framework for preference queries over sets, and designed a query rewriting technique based on superpreference and a consolidation technique based on $M$-relation to effectively optimize the generation of the best subsets of the given relation. For future directions, we see more opportunities in query optimization, especially for non-additive features. Also, a set preference with low selectivity, e.g., $\varnothing$, often leads to a large result set. We therefore consider the set preference elicitation an important topic whose study will enrich the picture of set preferences. Moreover, if the result set is large, we can adopt additional set ranking or browsing techniques to facilitate the navigation of results. In addition, one should study the embedding of the best-subset generation into relational query languages. For example, the best subsets could be returned non-deterministically. Another direction is designing more expressive set preference languages and studying their expressive power. An interesting problem is how to relax the fixed cardinality constraint in our framework. As we have pointed out in Sec. V, a complete absence of the cardinality constraint is likely to lead to problematic semantics. However, it is possible to relax this constraint in some applications: we might want to work with subsets of cardinality within a range instead. The $M$-relation optimization will continue to work as it is independent of the fixed cardinality assumption. On the other hand, the current version of superpreference relies on the fact that all subsets in competition are of the same cardinality. Extensions are needed to accommodate the relaxed cardinality assumption.

\section{REFERENCES}

[1] S. Börzsönyi, D. Kossmann, and K. Stocker, "The skyline operator," in ICDE, 2001, pp. 421-430.

[2] W. Kießling, "Foundations of preferences in database systems," in $V L D B, 2002$, pp. 311-322.

[3] W. Kießling and G. Köstler, "Preference SQL - design, implementation, experiences," in $V L D B, 2002$, pp. 990-1001.

[4] J. Chomicki, "Preference formulas in relational queries," ACM Trans. Database Syst., vol. 28, no. 4, pp. 427-466, 2003.

[5] C. Boutilier, R. I. Brafman, C. Domshlak, H. H. Hoos, and D. Poole, "CP-nets: A tool for representing and reasoning with conditional ceteris paribus preference statements," J. Artif. Intell. Res. (JAIR), vol. 21, pp. 135-191, 2004.

[6] S. Guha, D. Gunopulos, N. Koudas, D. Srivastava, and M. Vlachos, "Efficient approximation of optimization queries under parametric aggregation constraints," in $V L D B, 2003$, pp. 778-789.

[7] M. desJardins and K. Wagstaff, "DD-pref: A language for expressing preferences over sets," in AAAI, 2005, pp. 620-626.

[8] M. Binshtok, R. I. Brafman, S. E. Shimony, A. Mani, and C. Boutilier, "Computing optimal subsets," in AAAI, 2007, pp. 1231-1236.

[9] D. Mindolin and J. Chomicki, "Discovering relative importance of skyline attributes," in $V L D B, 2009$, pp. 610-621.

[10] J. Chomicki, P. Godfrey, J. Gryz, and D. Liang, "Skyline with presorting: Theory and optimizations," in Intelligent Information Systems, 2005, pp. 595-604.

[11] D. L. Kreher and D. R. Stinson, Combinatorial algorithms: generation, enumeration and search. CRC Press LTC, 1998.

[12] R. I. Brafman, C. Domshlak, and S. E. Shimony, "On graphical modeling of preference and importance," J. Artif. Intell. Res. (JAIR), vol. 25, pp. 389-424, 2006. 